\title{
Modelling the Near-IR Spectra of Red Supergiant-dominated Populations
}

\author{
Ariane Lançon ${ }^{1}$, Jay S. Gallagher ${ }^{2}$, Richard de Grijs ${ }^{3}$, \\ Peter Hauschildt $^{4}$, Djazia Ladjal ${ }^{5}$, Mustapha Mouhcine ${ }^{6}$, \\ Linda J. Smith ${ }^{7}$, Peter R. Wood ${ }^{8}$ and Natascha Förster Schreiber ${ }^{9}$ \\ ${ }^{1}$ Observatoire de Strasbourg (UMR 7550), 11 rue de l'Université, 67000 Strasbourg, France \\ email: lancon@astro.u-strasbg.fr \\ ${ }^{2}$ Dept. of Astronomy, 5534 Sterling, University of Wisconsin, Madison, WI 53706, USA \\ ${ }^{3}$ Dept. of Physics \& Astronomy, University of Sheffield, Hicks Building, Honusfield Rd., \\ Sheffield S3 7RH, UK \\ ${ }^{4}$ Hamburger Sternwarte, Gojenbergsweg 112, 21029 Hamburg, Germany \\ ${ }^{5}$ Institute of Astronomy, Katholieke Universiteit, Celestijnenlaan 200 B, 3001 Leuven, Belgium \\ ${ }^{6}$ Astrophysics Research Institute, Liverpool John Moores University, Twelve Quays House, \\ Egerton Wharf, Birkenhead, CH41 1LD, UK \\ ${ }^{7}$ Space Telescope Science Institute, 3700 San Martin Drive, Baltimore, MD 21218, USA \\ ${ }^{8}$ RSAA, Mt Stromlo Observatory, Cotter Road, Weston Creek, ACT 2611, Australia \\ ${ }^{9}$ MPI für Extraterrestrische Physik, Giessenbachstrasse, 85741 Garching, Germany
}

\begin{abstract}
We report on recent progress in the modelling of the near-IR spectra of young stellar populations, i.e. populations in which red supergiants (RSGs) are dominant. First, we discuss the determination of fundamental parameters of RSGs from PHOENIX model fits to their near-IR spectra; RSG-specific surface abundances are accounted for and effects of the microturbulence parameter are explored. New population synthesis predictions are then described and, as an example, it is shown that the spectra of young star clusters in M 82 can be reproduced very well from 0.5 to $2.4 \mu \mathrm{m}$. We warn of remaining uncertainties in cluster ages.
\end{abstract}

Keywords. galaxies: stellar content, galaxies: starburst, galaxies: star clusters, galaxies: individual (M82), infrared: galaxies, infrared: stars, stars: supergiants

\section{Introduction}

Red supergiant stars (RSGs) provide most of the near-IR light emitted by young stellar populations, such as those in starburst galaxies. As star forming environments tend to be dusty, rest-frame optical analyses are incomplete (highly obscured populations are missed) and it is crucial to improve our understanding of spectra at longer wavelengths. In the past, the near-IR analysis of young stellar populations has often been restricted to the determination of the average properties of the dominant stars, such as their spectral types or abundances. The subsequent interpretation of these results in terms of precise stellar population ages and star formation histories remains an enormous challenge, as it requires (i) a good understanding of the near-IR spectra of individual RSGs and (ii) adequate stellar evolution tracks. We have started a programme that aims at providing state of the art predictions for the emission of RSG-dominated populations and at characterizing remaining uncertainties. Currently, the project focuses on wavelengths between 0.81 and $2.4 \mu \mathrm{m}$ and spectral resolutions of order $\lambda / \delta \lambda=10^{3}$. 


\section{Empirical and synthetic spectra of red supergiants}

In principle, synthetic stellar spectra are more practical for the prediction of galaxy spectra than empirical ones, because theory allows us to sample parameter space without biases. Lançon et al. (2007) show that modern theoretical spectra can reproduce the nearIR (+optical) emission of giant stars well down to effective temperatures $T_{\text {eff }} \simeq 3400 \mathrm{~K}$, but that they are not yet satisfactory at lower temperatures and higher luminosities. They used new PHOEnIX models to compute spectra at the necessary resolution $(0.1 \AA$ before smoothing), with solar abundances and with the RSG-specific abundances obtained as the result of internal mixing along stellar evolution tracks; the models include some $10^{9}$ individual molecular and atomic lines, assume spherical symmetry, and allow dust to form if conditions are fulfilled. Model limitations include the assumptions of local thermal equilibrium (LTE) and of hydrostatic equilibrium. A sample of 101 empirical spectra covering wavelengths between $0.51,0.81$ or $0.90 \mu \mathrm{m}$ and $2.4 \mu \mathrm{m}$ was used for comparison (Lançon \& Wood 2000, Lançon et al. in preparation). The data were acquired with CASPIR on the $2.3 \mathrm{~m}$ ANU Telescope at Siding Spring and with SpeX at IRTF, Hawaii. Below $\mathrm{T}_{\text {eff }} \sim 3400 \mathrm{~K}$, uncertain input line lists are a problem in the models (especially for molecular bands around $1 \mu \mathrm{m}$ ). At high luminosity (luminosity class Ia and Iab), the main difficulty is to reproduce simultaneously extremely deep CN bands and the relative strengths of the CO bandheads around $1.7 \mu \mathrm{m}$ and at $2.3 \mu \mathrm{m}$. RSG-specific abundances improve fits to the CN bands. Exploratory calculations show that values near $10 \mathrm{~km} / \mathrm{s}$ for the "microturbulence" (a 1D-model parameter that hides poorly understood 3D physical phenomena) may be able to solve both problems (Fig. 1, top left). The calculation of a new grid has been launched to explore this further. In the mean time, the study shows that the population synthesis community still has to rely on empirical spectra for RSGs, and it warns that the lack of satisfactory stellar models implies large uncertainties on the derived fundamental parameters of the observed stars.

\section{Population synthesis using averaged stellar spectra}

In order to compute spectra of synthetic populations, we have constructed three sequences of average empirical spectra, corresponding to luminosity classes Ia, Iab and $\mathrm{Ib} / \mathrm{II}$. Each subset was sorted into bins according to the estimated $\mathrm{T}_{\text {eff }}$, the spectra were dereddened (an estimate of the reddening is provided by the model fits), and averages were computed. The sequences shown in Fig. 1 (top right) account for varying microturbulence in a preliminary way, based on the limited number of high microturbulence models available to us at the time of this writing. We chose to flag any star with an initial mass above $7 \mathrm{M}_{\odot}$ as a supergiant, which implies that the new spectra affect predictions up to the age of about $75 \mathrm{Myr}$ (Fig. 1, middle left). We note that predictions vary significantly depending on the adopted evolutionary tracks; different authors predict different red supergiant lifetimes, and main sequence rotation affects both the surface abundances and the final red (and blue) supergiant numbers.

\section{Star clusters in M82}

The synthetic spectra of single stellar populations (SSPs) at solar metallicity are compared with those of young star clusters in starbursts, such as M 82-L and M 82-F (Smith \& Gallagher 1999). The selected clusters are massive (well above $10^{5} \mathrm{M}_{\odot}$ ), i.e. stochastic effects due to an underpopulated RSG-branch are avoided. A few have well determined optical ages (based on standard non-rotating evolutionary tracks). Figure 1 (middle right 

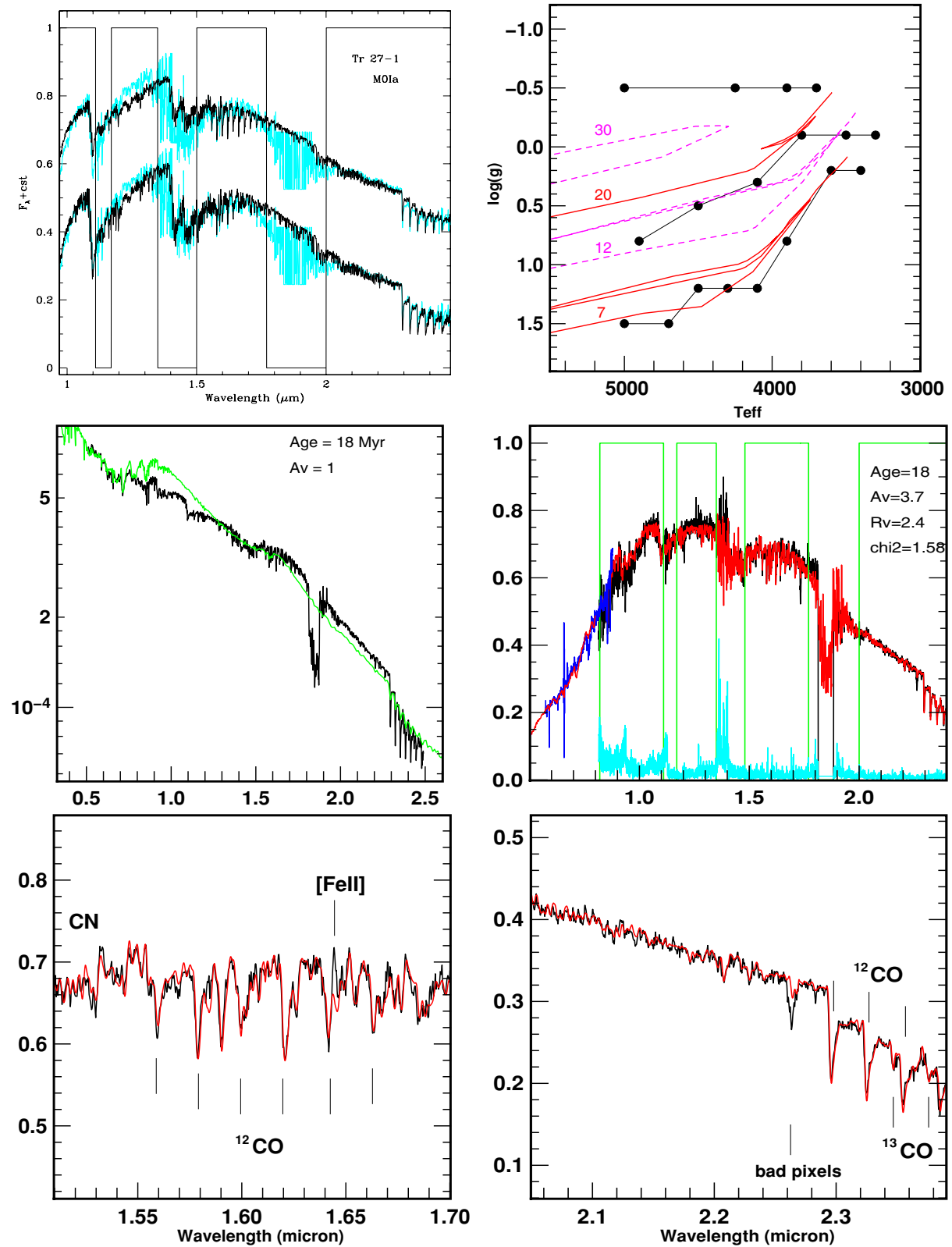

Figure 1. Top left: Spectrum of an MOIa RSG compared with models with $v_{\text {microturb }}=2 \mathrm{~km} / \mathrm{s}$ (top: $4200 \mathrm{~K}, \log (\mathrm{g})=-1, \mathrm{~A}_{V}=4.4$ ) and with $\mathrm{v}_{\text {microturb }}=10 \mathrm{~km} / \mathrm{s}$ (bottom: $4500 \mathrm{~K}, \log (\mathrm{g})=0$, $\mathrm{A}_{V}=4.7$; note the improved $\mathrm{CN}$ at $1.1 \mu \mathrm{m}$ and $\mathrm{CO}$ around 1.6 and $\left.2.3 \mu \mathrm{m}\right)$. Top right: Parameters assigned to the new sequences of average spectra, superimposed on the solar metallicity tracks of Bressan et al. (1993). Middle left: Comparison of a new SSP spectrum (black) with the standard predictions of Pegase.2 (differences are largest between 10 and $20 \mathrm{Myr}$ ). Middle right: Best near-IR fit to the spectrum of cluster M82-L. The extinction law of Cardelli et al. (1989) with $\mathrm{R}_{V}=2.4$ allows us to also reproduce the optical spectrum (from Smith \& Gallagher 1999). The error spectrum and the $\chi^{2}$ weighting function are shown. Bottom: Zoom-ins of the $\mathrm{H}$ and $\mathrm{K}$ windows. 
and bottom) shows cluster L, the cluster observed with SpeX with the best signal-tonoise ratio: an excellent fit is obtained over the whole available range in wavelength. Such results make the new models valuable tools for purposes such as weak emission line measurements. The $\chi^{2}$-test restricted to near-IR wavelengths not affected by strong telluric absorption shows that age is formally determined to an accuracy of about $\pm 10 \mathrm{Myr}$. Because of strong reddening, the optical age of cluster $\mathrm{L}$ cannot be determined as well as that of cluster F: 50-70 Myr (Gallagher \& Smith 2001, McCrady et al. 2005, Bastian et al. 2007). For F, our current models provide a near-IR age range of 32 to $46 \mathrm{Myr}$. This small but nevertheless significant disagreement calls for several comments. (i) Before accounting for luminosity-dependent microturbulence, we found a near-IR age of 10 to $20 \mathrm{Myr}$; we hope that our next generation of synthetic stellar spectra will significantly reduce uncertainties originating in uncertain fundamental parameters of stars. (ii) The spectrum used for optical age-dating and our near-IR spectrum have different slopes in the region of overlap. This suggests slightly different positions were observed: the obscuration across M82-F is not at all uniform. In addition, a younger cluster located at very small projected distance might contaminate the near-IR data. (iii) Modified stellar tracks (e.g. including rotation) might affect optical ages as well as near-IR ones.

\section{Conclusions}

The spectra of young stellar populations at solar metallicity, observed at $\mathrm{R} \sim 10^{3}$, can now be modelled well from the optical through the near-IR. Nevertheless, ages based on near-IR spectra remain severely affected by uncertainties. They are due mainly to systematic errors, which further work needs to characterize and reduce. Errors are associated on one hand with the fundamental parameters of red supergiant stars (theoretical spectra, microturbulence, surface abundances of $\mathrm{C}, \mathrm{N}$ and $\mathrm{O}$, non-LTE, variability, winds, giantsupergiant transition), and on the other with evolutionary tracks (convection, opacities, rotation, binarity, effects of a dense environment). We expect rapid progress in stellar atmosphere models to provide us with tools to test stellar tracks further. Complete optical and near-IR spectra of massive clusters such as those of M82 are useful test cases for the identification and correction of systematic errors, but even they are not trivial to exploit (due to inhomogeneous background populations and extinction, mass segregation, etc.).

\section{References}

Bastian, N., Konstantopoulos, I., Smith, L.J. \& Gallagher, J.S. 2007, MNRAS in press

Cardelli, J.A., Clayton, G.C. \& Mathis, J.S. 1989 ApJ 345, 245

Gallagher, J.S. \& Smith, L.J. 1999 MNRAS 304, 540

Lançon, A. \& Wood, P.R. 2000, A\&SAS 146, 217

Lançon, A., Hauschildt, P., Ladjal, D. \& Mouhcine, M. 2007, A\& $A$ in press

McCrady, N., Graham, J.R. \& Vacca, W.D. 2005 ApJ 621, 278

Smith, L. J. \& Gallagher, J. S. 2001 MNRAS 326, 1027

\section{Discussion}

Gustafsson: Do the models with high microturbulence include turbulent pressure in a consistent way?

LANÇON (AFter Discussion With P.H. AND H. LAmers): No. But the microturbulent velocities required to reproduce the spectra with $1 \mathrm{D}$ models are supersonic, which suggests that the actual process is not microturbulence... Therefore it is unclear how to relate this parameter of $1 \mathrm{D}$ models to pressure. 